Design of Optical Fibre Amplifiers

J.E.Townsend and R.I.Laming

Optoelectronics Research Centre,

The University of Southampton,

SO9 5NH, U.K.

\title{
Introduction
}

During the $1980^{\prime} s$ there has been increasing interest in rare-earth doped, single mode fibres for devices and sensors, of which the most exciting results have been achieved with erbium doped fibre amplifiers (EDFA). Great progress has been made since the first EDFA was reported by Mears in 1987 [1]. A gain of 26dB was achieved whereas $46.5 \mathrm{~dB}$ has now been demonstrated [2]. High speed, direct detection in fibre based transmission systems has allowed bit rates and transmission distances to be markedly increased. For example, use of twelve $1.48 \mu \mathrm{m}$ laser diode pumped EDFAs as power amplifiers has allowed transmission of $1.2 \mathrm{Gbit} / \mathrm{s}$ over more than $900 \mathrm{~km}$ of fibre [3]. The power penalty arising from dispersion and the presence of the EDFAs was only $0.6 \mathrm{~dB}$ at $10^{-9} \mathrm{BER}$, with no observed error floor due to accumulated noise. A number of similar systems have also been demonstrated with alternative pump sources and device configurations $[4,5]$. Thus the compatibility of EDFAs with long-haul communications systems has clearly been demonstrated. However, simultaneous improvement in manufacture and design of doped fibres has been essential. Here, the techniques for manufacture of such fibres are described and design criteria for optimal performance outlined. First, however, the advantages of EDFAs are summarised.

All-optical amplifiers are attractive in fibre based communications networks for low maintenance and faster operating rates than conventional electronic repeaters. If amplification is achieved 'on line' by incorporating the amplifier into standard communications grade fibre then interface losses are reduced to negligible levels. Further, the geometry of optical fibres, with small waveguiding area and long interaction lengths allows efficient interaction of light with the dopant so that low power semiconductor lasers may be used to excite the active ions. A dopant is therefore required which can be incorporated into the fibre and is able to amplify signals at the chosen system wavelength, either $1.3 \mu \mathrm{m}$ or near $1.55 \mu \mathrm{m}$. $\mathrm{Er}^{3+}$ ions fluoresce around $1.55 \mu \mathrm{m}$ when excited by optical pumping at one of a number wavelengths, as shown in figure 1 [6]. With reference to the figure it may be seen that emission occurs from the ${ }^{4} I_{13 / 2}$ level to the ground state ie. 3 level operation. Thus, a population inversion is essential to bleach out the optical loss at the emission frequency before gain can be achieved - a problem in bulk materials but easily overcome in the fibre geometry.

\section{Fabrication methods}

Conventional manufacturing processes have been modified to incorporate erbium ions into standard fibres. Optical fibres are drawn, or pulled from preforms whose characteristics determine those of the fibre. Two processes are widely employed to prepare single mode or graded index preforms for telecommunications applications. The Modified Chemical Vapour Deposition process (MCVD) was proposed in the USA [7] and England [8] in the early 1970's and relies on gas phase oxidation of vapour phase reagents to form the glass. The Vapour Axial Deposition method relies on 
hydrolysis of the same materials and was developed approximately 10 years ago by the Japanese [9]. The MCVD process is described here, with the modifications required to incorporate rare-earth dopants such as $\mathrm{Er}^{3+}$.

MCVD

MCVD is based on the high temperature oxidation of reactant gases inside a rotating tube which is heated by an external source. A schematic of the process is shown in figure $2[10]$. Controlled quantities of the dopants are transported to the reaction zone by passing gases (e.g. $\mathrm{O}_{2}$ ) through the liquid precursors, usually halides, thereby avoiding contamination from transition metal ion impurities. Silicon tetrachloride $\left(\mathrm{SiCl}_{4}\right)$ is used to form silica, the main component of optical fibres. $\mathrm{SiO}_{2}$ is attractive due to the low losses and low dispersion in the region $1300 \mathrm{~nm}$ to $1550 \mathrm{~nm}$ as well as its mechanical and chemical strength. $\mathrm{GeO}_{2}$ and $\mathrm{P}_{2} \mathrm{O}_{5}$ are commonly added as fibre core material to raise the refractive index of silica. Conversely, $\mathrm{B}_{2} \mathrm{O}_{3}$ or fluorine reduce the refractive index of the deposited glass.

The reactant gasses flow, with $\mathrm{O}_{2}, \mathrm{~N}_{2}$ and He, inside a rotating silica substrate tube mounted on a glass working lathe. The tube is heated by an external burner traversing in the same direction as the gas flow. Gas phase oxidation in the burner's hot zone (typically $1700^{\circ} \mathrm{C}$ ) results in the formation of oxide soot particles, which is deposited further downstream. As the burner passes over the deposited layer, it is fused to a glass by viscous sintering. Deposition temperatures depend on glass composition and are sufficiently high to sinter the deposited material but not so high as to cause distortion of the tube (usually between $1300^{\circ} \mathrm{C}$ and $1650^{\circ} \mathrm{C}$ ). Many layers of defined index are built up in this manner. After deposition of the required amount of material, the tube is collapsed to a solid rod by heating to approximately $2000^{\circ} \mathrm{C}$, sufficient to soften the glass and allow collapse under surface tension.

Fibre is drawn from the preform by heating the rod and, in effect, stretching it to the required diameter (usually $125 \mu \mathrm{m}$ ) under controlled conditions. During the process the fibre is coated with a plastic for protection.

\section{Fabrication of rare-earth doped fibres}

Several techniques for incorporating rare-earth ions have been developed, suitable for both VAD and MCVD. High vapour pressure organometallic reagents have been employed [11] in conventional MCVD, with the rare-earth ion being transported in the vapour phase to the reaction zone in the same manner as the glass forming material. The technique is simple to implement but the precursors are toxic, not widely available and very expensive.

An alternative, and widely used, vapour phase process was developed at Southampton University, to exploit the high purity achievable via the vapour phase, without needing novel reagents. Solid, low vapour pressure rare-earth halide precursors are used [12] and incorporated in the fabrication process as shown in figure 3. Rare-earth halide precursor is located in a dopant chamber, upstream of the substrate tube. During conventional deposition of the core glass the dopant chamber is heated to approximately $1000^{\circ} \mathrm{C}$ to achieve a significant vapour pressure of rare-earth halide. Accurate temperature control is essential for control of precursor vapour pressure and hence dopant concentration. The vapour is carried to the reaction zone with the glass forming regents where it is oxidised and deposited as normal. Collapse and fibre drawing are conventional. 
Fibres containing a few hundred parts per million (ppm) are readily prepared in this manner. Losses approach those of telecommunications fibres, as shown in figure 4, for a fibre doped with approximately $100 \mathrm{ppm}$ of $\mathrm{Er}^{3+}$. Away from the dopant absorption bands losses of order $5 \mathrm{~dB} / \mathrm{km}$ are commonplace [13]. Further, backscatter measurements [14] show that the dopant is incorporated evenly in the glass. However, this method is not very versatile since it is difficult to add either high dopant concentrations or incorporate several types of ion simultaneously in predetermined ratios.

More recently, a novel method, whereby the ions are added from solution, has been developed at Southampton University [15]. The process, described in figure 5 overcomes the difficulties previously encountered. Although there is an increased risk of impurity contamination via the liquid phase, low loss fibres with high dopant concentrations are fabricated. Very simple modifications to the MCVD process allow the incorporation of dopant ions via solution. The conventional fabrication process is followed until the core layer is deposited. Here, a reduction in burner temperature of around $500^{\circ} \mathrm{C}$ allows the formation and deposition of the oxide soot without subsequent fusion of this porous layer. The tube is then removed from the lathe and the porous frit immersed in a dilute solution of the dopant ions. Additional glass modifying reagents can also be added at this stage, for example alumina can be included from $\mathrm{AlCl}_{3}$ in solution. Water and alcohols are commonly used as solvents since they are safe to handle and the halide precursors are soluble in both. After soaking, excess solution is removed, the tube replaced in the lathe and the porous layer dried. Dehydration is based on the VAD method [16]. $\mathrm{Cl}_{2}$ is passed through the inside of the tube which is heated to approximately $1000^{\circ} \mathrm{C}$, so evaporating residual solvent. As a result, hyroxyl ion contamination is insignificant, with residual concentrations of around $0.25 \mathrm{ppm}$. Dopant ions are not volatile at this temperature and remained trapped in the frit. Fusion of the soot layer occurs at the temperature conventionally employed and collapse of the tube into the preform is also standard.

\section{Amplifier design parameters}

A number of parameters affect the design and performance of EDFAs, including host material, dopant concentration and pump wavelength. The importance of each of these on fibre design is discussed here.

Host glass

Telecommunications grade fibres typically comprise around 908 silica, with the remainder being compatible glass forming material of similar structure to control the refractive index. Although the spectral characteristics of the rareearth ions are sensitive to surrounding ligand field (i.e. host), it might be anticipated that the spectral features are unlikely to be affected by such small changes in host composition. Nevertheless, the addition of a few percent of index modifying ions is found to be sufficient to alter the fluorescence spectra of dopant ions.

In addition to host effects, however, the gain spectrum is highly dependent on the relative populations of the metastable and ground states (hence pump power) [6], because $\mathrm{Er}^{3+}$ operates as a 3 level system. Gain spectra for highly inverted germanosilicate and aluminosilicate $\mathrm{Er}^{3+}$ doped fibre are shown in figure 6 . Nonuniform emission spectra arise from Stark splitting of metastable and ground state 
energy levels, with the selection of levels being host dependent. In $\mathrm{GeO}_{2}-\mathrm{SiO}_{2}$, the gain spectrum is highly wavelength dependent, peaking at $1.535 \mu \mathrm{m}$. Typically, the $3 \mathrm{~dB}$ bandwidth is $-3 \mathrm{~nm}$ for a $30 \mathrm{~dB}$ amplifier. The peak gain cross section has been determined experimentally and is found to be higher in germanosilicate core EDFA's than those containing alumina. The peak gain is shifted to $1.531 \mu \mathrm{m}$ in $\mathrm{Al}_{2} \mathrm{O}_{3}-\mathrm{SiO}_{2}$ core fibre and, for a $30 \mathrm{~dB}$ amplifier, the $3 \mathrm{~dB}$ bandwidth is $7 \mathrm{~nm}$. However, with reference to the figure, it may be seen that there is a relatively constant gain region extending from approximately $1.538 \mu \mathrm{m}$ to $1.560 \mu \mathrm{m}$, where gains in excess of $20 \mathrm{~dB}$ have been demonstrated. Atkins [17] has exploited this region, under reduced inversion, to achieve a $25 \mathrm{~dB}$ gain amplifier with $3 \mathrm{~dB}$ bandwidth of $35 \mathrm{~nm}$. More recently however, a novel method for generating a broad band, spectrally flat gain spectrum has been demonstrated by Tachibana [18]. An essentially flat gain of $27 \mathrm{~dB}$ is obtained over $33 \mathrm{~nm}$ without loss of pump efficiency or saturation performance by incorporating an optical notch filter in the middle of the amplifier. The flattened gain spectrum is shown in figure 7. Thus, in general, $\mathrm{GeO}_{2}-\mathrm{SiO}_{2}$ core EDFAs are preferred for preamplifier applications, where high efficiency and gain cross sections are required whilst the $\mathrm{Al}_{2} \mathrm{O}_{3}-\mathrm{SiO}_{2}$ host is preferred in broad band systems .

$\mathrm{Er}^{3+}$ suffers parasitic, or excited state, absorption (ESA) from the metastable energy level at several wavelengths. A significant ESA cross section around potential pump energies or the signal band decreases the population inversion with increasing pump power and consequent reduction in performance. ESA is measured by comparing the spectral attenuation of an erbium doped fibre when pumped (i.e. under population inversion) and unpumped. Significant pump ESA is recorded at a number of potential pump wavelengths, as shown in figure 8 for an $\mathrm{Er}^{3+}$ doped $\mathrm{Al}_{2} \mathrm{O}_{3}-\mathrm{SiO}_{2}$ fibre. Bands around $488 \mathrm{~nm}, 514.5 \mathrm{~nm}, 655 \mathrm{~nm}$ and $810 \mathrm{~nm}$ are seriously affected. However, a strong host dependence is recorded, as shown in Table 1 [19], where the ratio of ESA and GSA cross sections is tabulated for a number of hosts. 532nm may be employed to pump EDFAs, but $980 \mathrm{~nm}$ is a more efficient pump wavelength. In-band pumping around $1485 \mathrm{~nm}$ is also employed [20]. Signal ESA is measured in a similar manner and no significant ESA is recorded near $1.55 \mu \mathrm{m}$.

Host composition also determines emission decay times of the fluorescence. Differences in the lattice vibration frequency (phonon energy) of $\mathrm{GeO}_{2}-\mathrm{SiO}_{2}$ and $\mathrm{Al}_{2} \mathrm{O}_{3}-\mathrm{SiO}_{2}$ hosts leads to lifetimes of around $12 \mathrm{~ms}$ and $-10 \mathrm{~ms}$ for low $\mathrm{Er}^{3+}$ concentrations in the two materials, respectively. This parameter alone does not determine quantum efficiency of an amplifier thus the shorter emission decay time in $\mathrm{Al}_{2} \mathrm{O}_{3}-\mathrm{SiO}_{2}$ is not a drawback.

\section{Dopant concentration}

Both fluorescence lifetime and quantum efficiency (QE) are affected by the ion concentration in the fibre. QE data give the clearer indication of amplifier performance but are difficult to measure directly. Data presented in figure 9 show the dependence of pump energy conversion efficiency on dopant concentration [21] and give an indication of QE. A $980 \mathrm{~nm}$ pump source was employed to generate the $1535 \mathrm{~nm}$ emission so 648 conversion efficiency corresponds to quantum limited operation (100\% photon conversion). A strong dependence on dopant concentration is recorded, particularly in $\mathrm{GeO}_{2}-\mathrm{SiO}_{2}$ for $\mathrm{Er}^{3+}$ concentrations in excess of $200 \mathrm{ppm}$, but the effect is alleviated by the addition of alumina to the host. The reduction in $\mathrm{QE}$ is attributed to a cooperative upconversion mechanism, described in figure 10 [22]. Energy transfer takes place between neighbouring ions, both excited to the 
metastable state $\left({ }^{4} I_{13 / 2}\right)$. The acceptor ion is promoted to the ${ }^{4} I_{9 / 2}$ level whilst the donor returns to the ground state. For the process to occur the ions must be located close to each other and such an effect is not expected at low dopant concentrations. However, rare-earth ions are only slightly soluble in $\mathrm{GeO}_{2}-\mathrm{SiO}_{2}$ glasses, tending to phase separate in the host, reducing average ion-ion separation. The slightly altered geometry of $\mathrm{Al}_{2} \mathrm{O}_{3}-\mathrm{SiO}_{2}$ glass allows the ions to redisperse. Nevertheless, a reduction in $\mathrm{QE}$ also seems to occur in $\mathrm{Al}_{2} \mathrm{O}_{3}-\mathrm{SiO}_{2} \mathrm{EDFAs}$, around $800 \mathrm{ppm}$. In summary, the preferred dopant concentration is low, around $100 \mathrm{ppm}$ for efficient EDFAs based on $\mathrm{GeO}_{2}-\mathrm{SiO}_{2}$ and a few hundred ppm in $\mathrm{Al}_{2} \mathrm{O}_{3}-\mathrm{SiO}_{2}$ core fibres.

The limit on dopant concentration determines the amplifier length, owing to the contribution to gain from each ion. The ratio of absorption and emission cross sections has been determined as 1.2 to 1 at the absorption peak, for both alumina and germania doped silica based hosts. Further, the absorption per unit ion is around $0.05 \mathrm{~dB} \cdot \mathrm{m}^{-1} \cdot \mathrm{ppm}^{-1}$ at $1535 \mathrm{~nm}$ in a multimoded silica based fibre. Hence, to achieve $30 \mathrm{~dB}$ gain from a finite pump power, $50 \mathrm{~dB}$ absorption at $1535 \mathrm{~nm}$ is typically employed, which, given the concentration limit of $100 \mathrm{ppm}$, defines a minimum amplifier length of $10 \mathrm{~m}$.

\section{Dopant distribution}

The measured absorption in a length of $\mathrm{Er}^{3+}$ doped fibre is a function of the overlap between dopant and trnsmitted light as well as of concentration. First, the distribution of dopant in $\mathrm{GeO}_{2}-\mathrm{SiO}_{2}$ core fibres does not follow a perfect step index and second, the modal overlap between dopant and pump or signal power is poor in a single mode fibre. The significance of these two features is discussed separately here.

The distribution of $\mathrm{Er}^{3+}$ in $\mathrm{GeO}_{2}-\mathrm{SiO}_{2}$ fibre prepared by MCVD follows that of $\mathrm{GeO}_{2}$, with a lower concentration of dopant in the centre of the fibre, as shown in figure 11. The distribution arises from evaporation of germania during the collapse stage of the MCVD process. $\mathrm{Er}^{3+}$ is assumed to be attached to the volatile component and is therefore, also evaporated. Conversely, in $\mathrm{Al}_{2} \mathrm{O}_{3}-\mathrm{SiO}_{2}$ fibres no loss of $\mathrm{Al}_{2} \mathrm{O}_{3}$ or $\mathrm{Er}^{3+}$ occurs, as seen in figure 12. Thus, much of the light propagating along the germanosilicate core fibre will not interact with the dopant. The "effective" or measured dopant concentration is approximately half the true or local concentration, as determined by materials analysis.

Further, in single mode fibres, the overlap between dopant and pump mode is incomplete whilst as little as 508 of the signal power may propagate in the core of a fibre which is single mode around $950 \mathrm{~nm}$. The 3-level nature of $\mathrm{Er}^{3+}$ necessitates pumping half the ions from the ground to the metastable state to achieve gain. It is therefore desirable to locate the dopant where the pump intensity is highest, and to prevent there being any ions in regions of low pump power where a population inversion may not be obtained. Thus, the dopant should be confined to the region of highest power density for efficient operation of an EDFA, ie. the centre of the core [23]. The single mode signal beam also has its maximum intensity on the axis and therefore overlaps well with the confined excited ions. A reduced bleaching power (that required to just achieve an inversion) has been confirmed in confined EDFAs. Alumina is usually employed as a codopant in such a configuration to minimise loss of dopant from the centre and to allow higher dopant concentrations to be employed. A core occupancy of around $50 \%$ is effective in $980 \mathrm{~nm}$ 
pumped systems. An alternative approach, particularly suitable for in-band pumped systems, is to operate the EDFA well beyond cutoff, with only part on the power guided in the core region.

In both cases, the minimum amplifier length is increased by around 50\%, giving a practical value of around $20 \mathrm{~m}$ in $\mathrm{GeO}_{2}-\mathrm{SiO}_{2}$ fibres. Baseline losses (e.g. waveguide imperfections) may then become significant. Typical values of $5 * 10^{-3} \mathrm{~dB} / \mathrm{m}$ are recorded in doped $\mathrm{GeO}_{2}-\mathrm{SiO}_{2}$ fibres, giving an excess loss of $0.1 \mathrm{~dB}$ per EDFA an acceptable value. High performance amplifiers of $160 \mathrm{~m}$ in length have been demonstrated. However, in $\mathrm{Al}_{2} \mathrm{O}_{3}-\mathrm{SiO}_{2}$ fibres, losses typically of order $0.05 \mathrm{~dB} / \mathrm{m}$, due to the incorporation of impurities such as $\mathrm{Fe}^{2+}$ in the doping process [13] although losses of $0.01 \mathrm{~dB} / \mathrm{m}$ are obtained by the author. The loss scales with concentration of alumina. Hence, the excess loss can be significant in an $\mathrm{Al}_{2} \mathrm{O}_{3}-\mathrm{SiO}_{2}$ EDFA more than $20 \mathrm{~m}$ long, and elevated dopant concentrations should be used.

Given that it is preferable to operate the pump in a single mode configuration constraints are placed on the geometry of the core, particularly if a $980 \mathrm{~nm}$ pump is employed. The modal parameters should be matched, at the signal wavelength, to dispersion matched telecommunications fibre. Also, the fibre should be single moded below $980 \mathrm{~nm}$. A high numerical aperture (around 0.2 ) is preferred for efficient pumping owing to the decreased core area and hence increased pump power density, although a core diameter in excess of $3 \mu \mathrm{m}$ is preferred for efficient launching. Further, the fibre waveguiding characteristics will be poor around $1550 \mathrm{~nm}$ unless a high numerical aperture is used. It is found that all these criteria are fulfilled in a fibre of numerical aperture around 0.2 with second mode cutoff near $970 \mathrm{~nm}$. The geometric requirements are relaxed for in-band pumping since the pump wavelength $(1485 \mathrm{~nm})$ is close to the signal wavelength $(1535 \mathrm{~nm})$, but as stated above shorter cutoff wavelengths are preferred for effectively confining the dopant.

\section{Summary}

In summary, for an optimum EDFA, low dopant concentrations, of order $100 \mathrm{ppm}$, should be employed in $\mathrm{GeO}_{2}-\mathrm{SiO}_{2}$ core fibres. Long lengths may be used owing to the low attenuation, similar to that of conventional fibres. Higher concentrations (a few hundred ppm) may be employed in $\mathrm{Al}_{2} \mathrm{O}_{3}-\mathrm{SiO}_{2}$ core fibres to reduce the amplifier length, owing to the excess loss introduced by $\mathrm{Al}_{2} \mathrm{O}_{3}$. A host dependent gain spectrum obtained, with higher cross sections (more gain per unit pump power) being recorded in $\mathrm{GeO}_{2}-\mathrm{SiO}_{2}$ fibres whilst the gain bandwidth is broader in $\mathrm{Al}_{2} \mathrm{O}_{3}-\mathrm{SiO}_{2}$ EDFAs. Two pump wavelengths are preferred, $980 \mathrm{~nm}$ or $1485 \mathrm{~nm}$, neither of which suffers ESA in either host. Fibres which are single mode at the pump wavelength are employed, with a high numerical aperture being essential in $980 \mathrm{~nm}$ pumped systems. For efficient pumping the dopant is either confined to the centre of the core or the EDFA operated well beyond cutoff.

\section{References}

1. R.J.Mears, L.Reekie, I.M.Jauncey and D.N.Payne, "High gain rare-earth doped fibre amplifier at $1.54 \mu \mathrm{m} "$, OFC '87, Reno, Nevada, Paper W12, 1987

2. Y.Kimura, K.Suzuki and M.Nakazawa, $446.5 \mathrm{~dB}$ gain in $\mathrm{Er}^{3+}$ doped fibres amplifier pumped by $1.48 \mu \mathrm{m}$ GaInAsP laser diodes", Electronics Letters, 
vol.25, pp.1656-1657, 1989

3. N.Edagawa, Y.Yoshida, H.Taga, S.Yamamoto, K.Mochizuki and H. Wakabayashi, "Non regenerative optical transmission experiment using $\mathrm{Er}^{3+}$ doped fibre amplifiers", ECOC'89, Gothenberg, Paper PD-8, 1989

4. S.Saito, T.Imai, T.Sugie, N.Ohkawa, Y.Ichihashi and T.Ito, "An over 2,200km coherent transmission experiment at $2.5 \mathrm{Gbit} / \mathrm{s}$ using erbium doped fibre amplifiers", OFC '90, San Fransisco, Paper PD2, 1990

5. R.Welter, R.I.Laming, R.S.Vodhanel, W.B.Sessa, M.W.Maeda and R.E.Wagner, "Performance of an erbium doped fibre amplifier in a 16-channel coherent broadcast network experiment" CLEO '89, Baltimore, postdeadline session, 1989

6. D.N.Payne and R.I.Laming, "Optical fibre amplifiers", OFC '90, San Francisco, USA, Tutorial pp.331-353, 1990

7. J.B.MacChesney and P.B.0'Connor, US Patent 4-217027

8. D.N.Payne and W.A.Gambling, "Optical waveguides", Patent 34813/74

9. T.Izawa, S.Kobayashi, S.Sudo and F.Hanawa, "Continuous fabrication of high silica fibre preform", IOOC '77, Tokyo, Paper C1-1, 1977

10. S.R.Nagel, J.B.MacChesney and K.L.Walker, An overview of the modified chemical vapour deposition process and performance", IEEE J. Quantum Electronics, vol.QE-18, pp.459-477, 1982

11. D.A.Thompson, P.L.Bocko and J.R.Gannon, "New source compounds for the fabrication of doped optical waveguide fibres", SPIE vol. 506 Fibre Optics in adverse environments II, pp. 170-175, 1984

12. S.B.Poole, D.N.Payne and M.E.Fermann, "Fabrication of low loss optical fibres containing rare-earth ions", Electronics Letters, vol.21, pp.737-738, 1985

13. S.P.Craig-Ryan, B.J.Ainslie and C.A.Millar, "Fabrication of long lengths of low excess loss erbium doped optical fibre", Electronics Letters, vol.26, pp.185-186, 1990

14. S.B.Poole, D.N.Payne, R.J.Mears, M.E.Fermann and R.I.Laming, "Fabrication and characterisation of low loss optical fibres containing rare-earth ions", J. Lightwave Technology, vol. LT-4, pp.870-876, 1986

15. J.E.Townsend, S.B.Poole and D.N.Payne, "Solution doping technique for the fabrication of rare-earth doped optical fibres", Electronics Letters, vol.23, pp.329-331, 1987

16. K.Sanada, R.Yamauchi, 0.Fukuda and $\mathrm{K}$. Inada, "Behaviour of $\mathrm{GeO}_{2}$ in dehydration and consolidation processes of the VAD method", OFC '84, pp.26-27, 1984 
17. C.G.Atkins, J.F.Massicott, J.R.Armitage, R.Wyatt, B.J.Ainslie and S.P.CraigRyan, High gain, broad spectral bandwidth erbium doped fibres amplifier pumped near $1.5 \mu \mathrm{m}$, Electronics Letters, vol.25, pp.910, 1989

18. M.Tachibana, R.I.Laming, P.R.Morkel and D.N.Payne, "Gain shaped erbium doped fibre amplifier (EDFA) with broad spectral bandwidth", Optical amplifiers and their applications, Montery, pp. 44-47, paper MD1, 1990

19. R.I.Laming, S.B.Poole and E.J.Tarbox, "Pump excited state absorption in Er doped fibres", Optics Letters, vol.13, pp.1084-1086, 1988

20. E.Snitzer, H.Po, F.Hakimi, R.Tumminelli and B.C.McCollum, "Erbium fibres laser at $1.55 \mu \mathrm{m}$ with pump at $1.49 \mu \mathrm{m}$ and $\mathrm{Yb}$ sensitised Er oscillator", OFC '88, paper PD2, 1988

21. R.I.Laming, D.N.Payne, F.Meli, G.Grasso and E.J.Tarbox, "Highly saturated erbium doped fibre power amplifiers", Optical amplifiers and their applications, Montery, 1990

22. B.J.Ainslie, S.P.Craig-Ryan, S.Davey, J.R.Armitage, C.G.Atkins and R.Wyatt, "Optical analysis of erbium doped fibres for efficient lasers and amplifiers", IOOC '89, Kobe, Japan, Paper 20A3-2, 1989

23. B.J.Ainslie, S.P.Craig-Ryan and J.R.Armitage, "Optical fibre with fluorescent additive", U.K. patent 88308450.1 


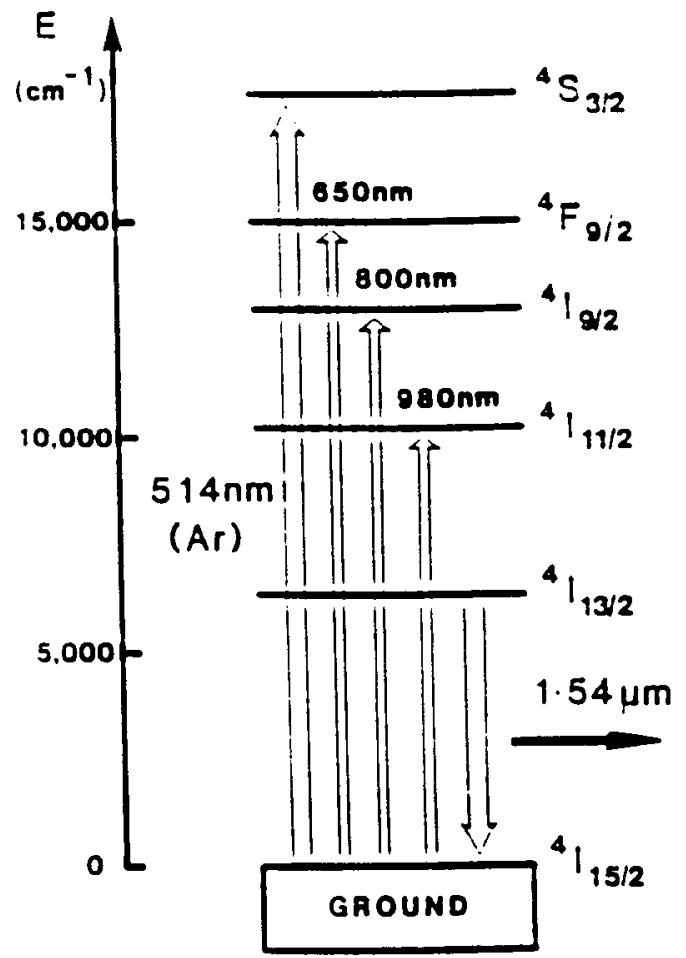

Figure 1. Energy level diagram of $\mathrm{Er}^{3+}$ showing possible pump wavelengths

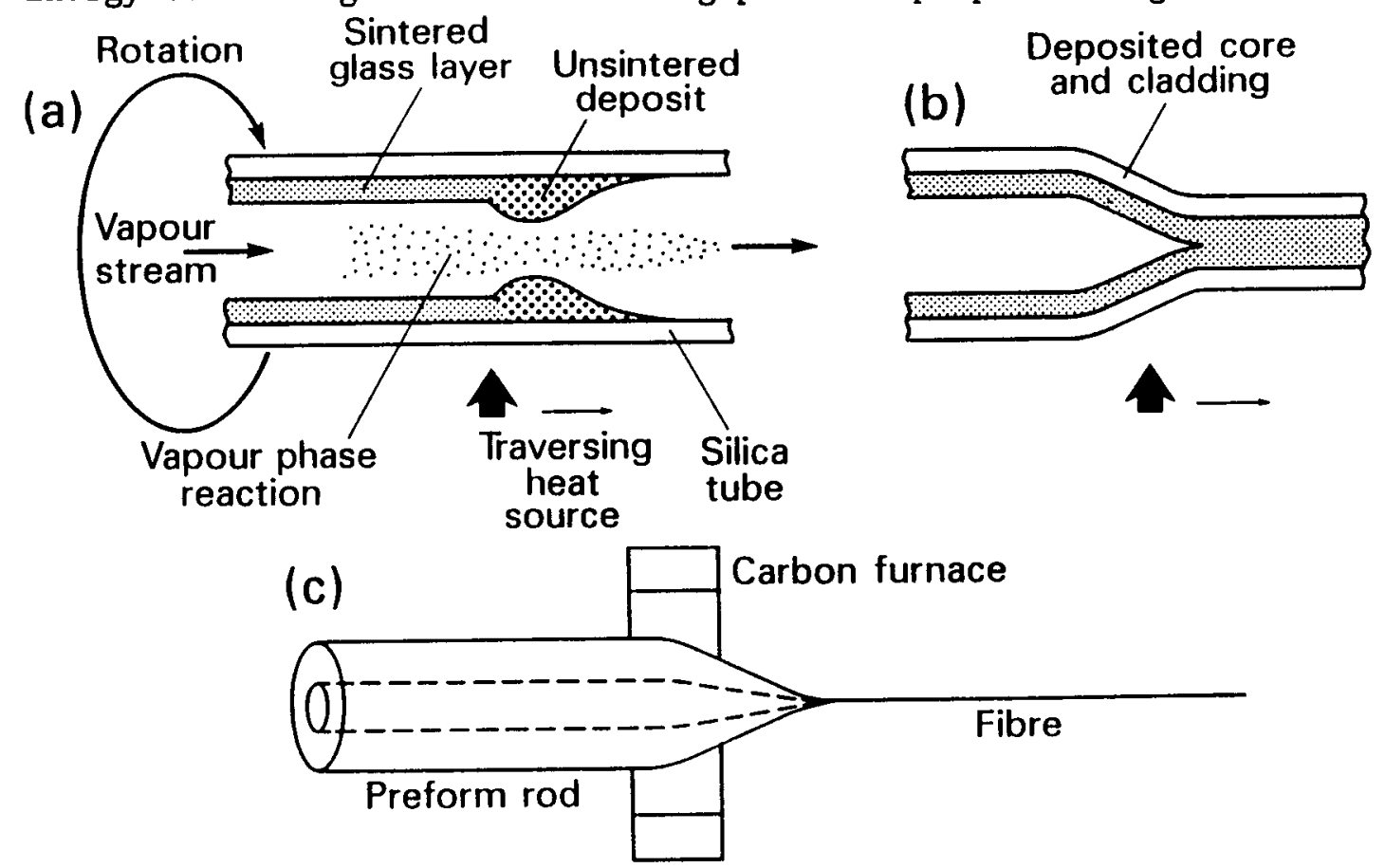

Figure 2. Schematic showing the MCVD method for the preparation of optical fibres;

a) deposition, b) collapse to produce a preform and c) fibre drawing 


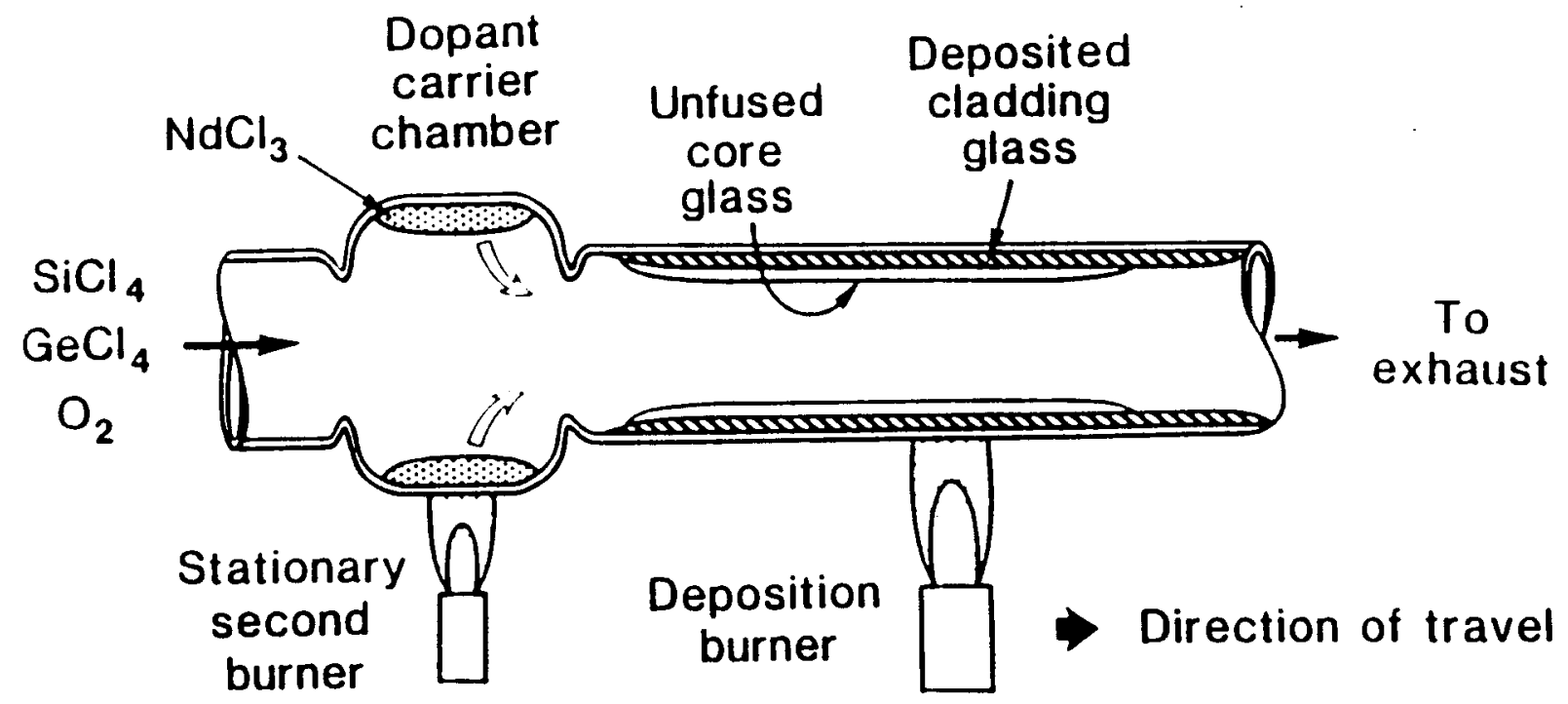

Figure 3. Vapour phase process for fabrication of rare-earth doped optical fibres

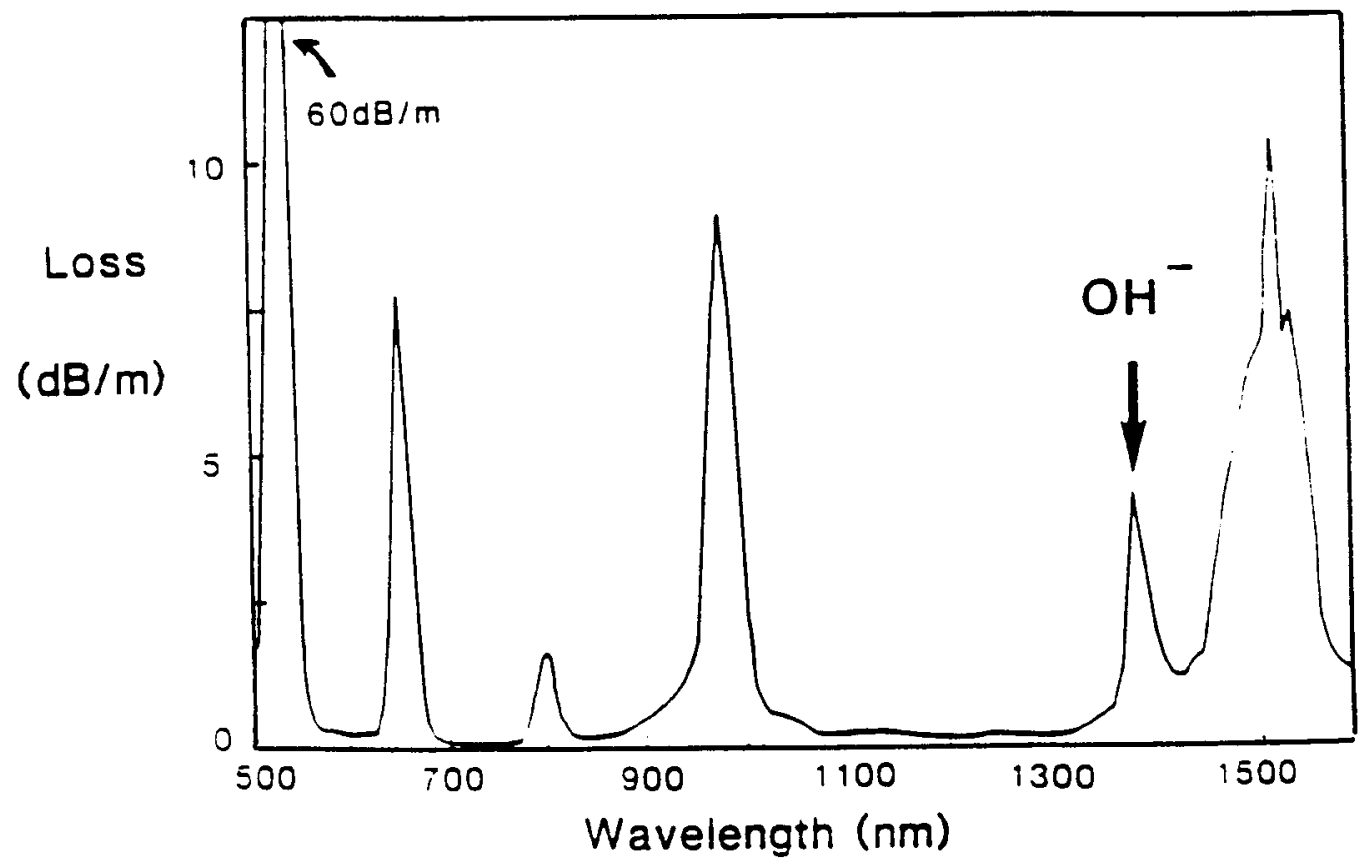

Figure 4. Absorption spectrum of optical fibre doped with $\operatorname{Er}^{3+}$ ions 


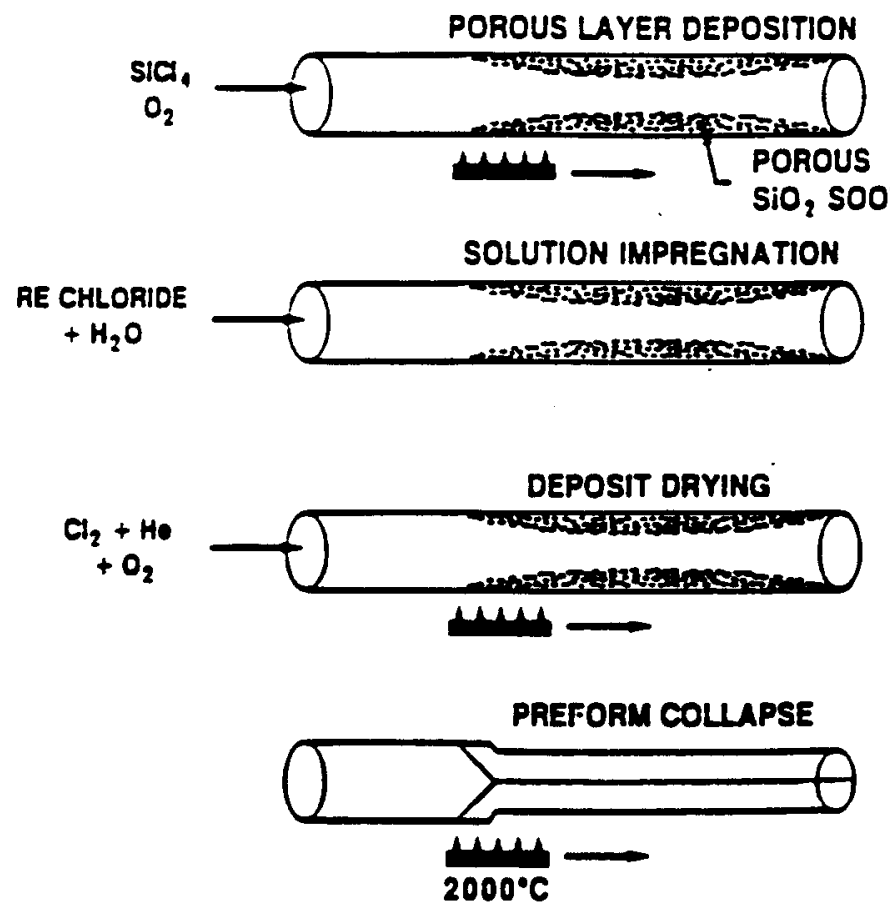

Figure 5. Schematic of the solution doping process for manufacture of rare-earth doped fibres via MCVD

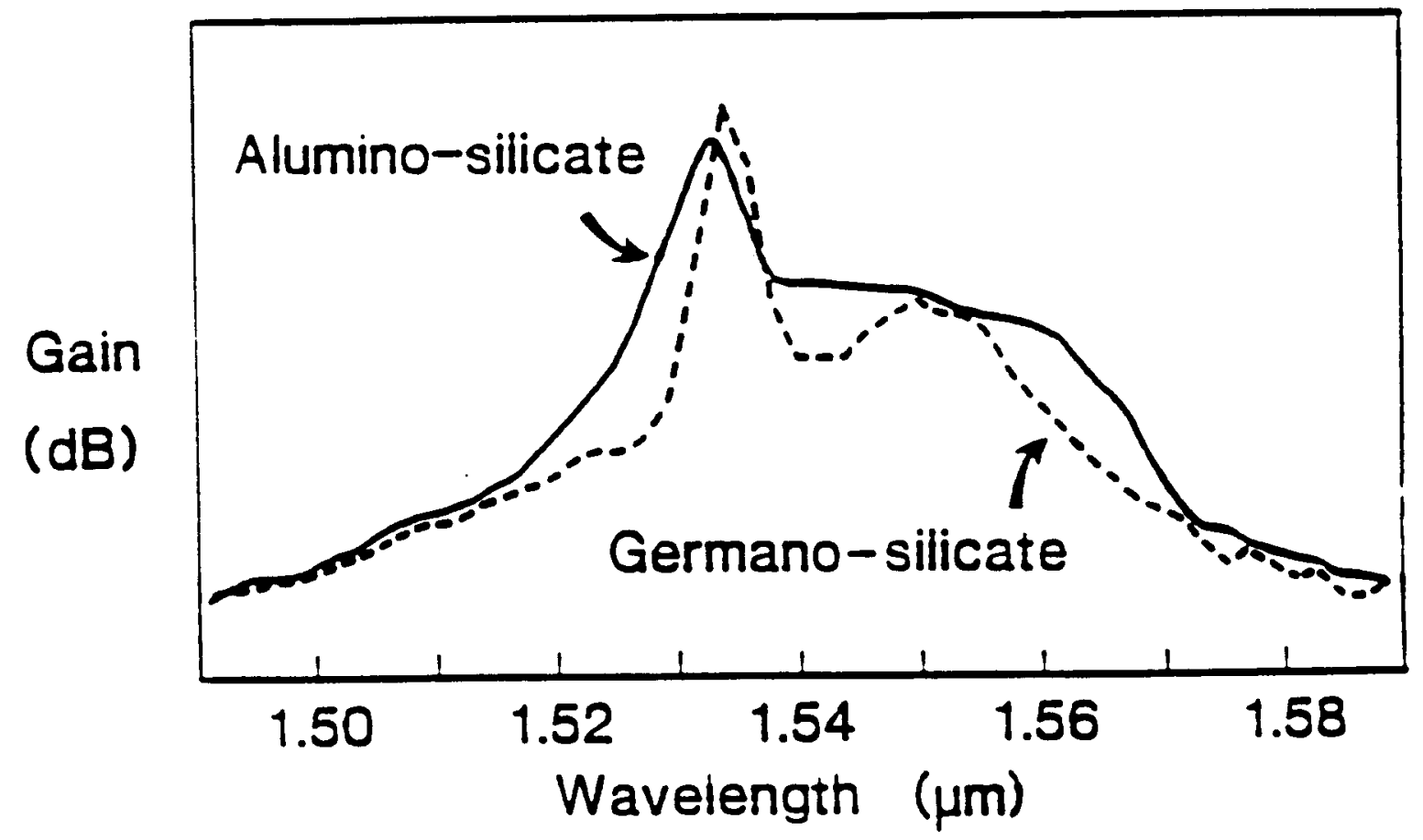

Figure 6. Gain spectrum of $\mathrm{Er}^{3+}$ ions in two optical fibre host glasses prepared by MCVD 


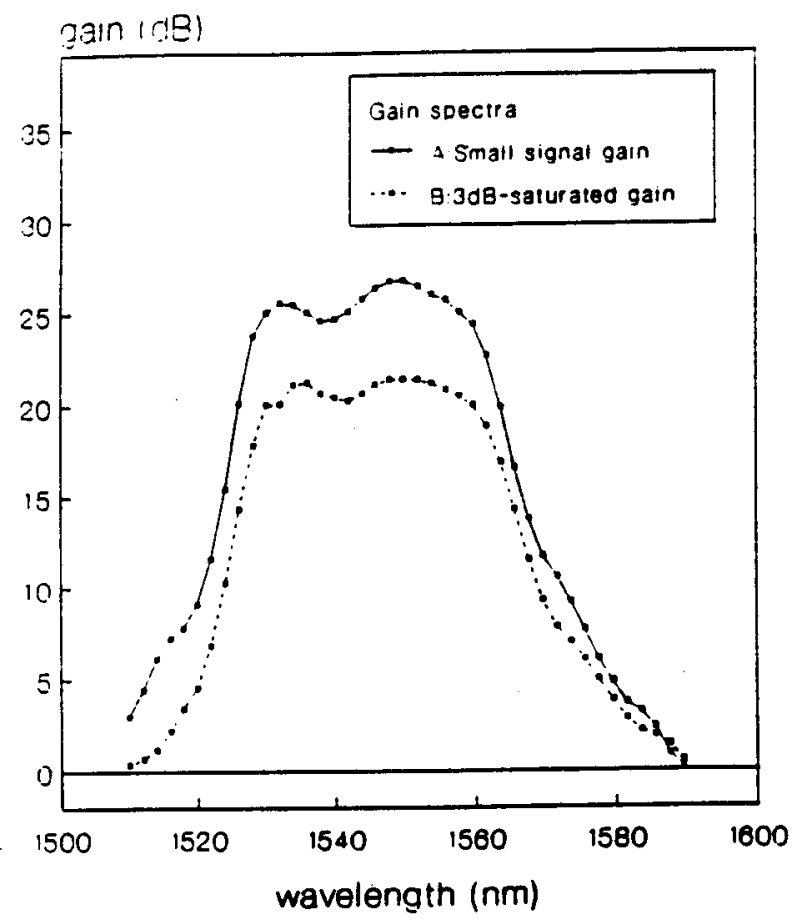

Figure 7. Flattened gain spectra for small signal and $3 \mathrm{~dB}$ saturated operation of EDFA, including optical notch filter (after Tachibana et al)

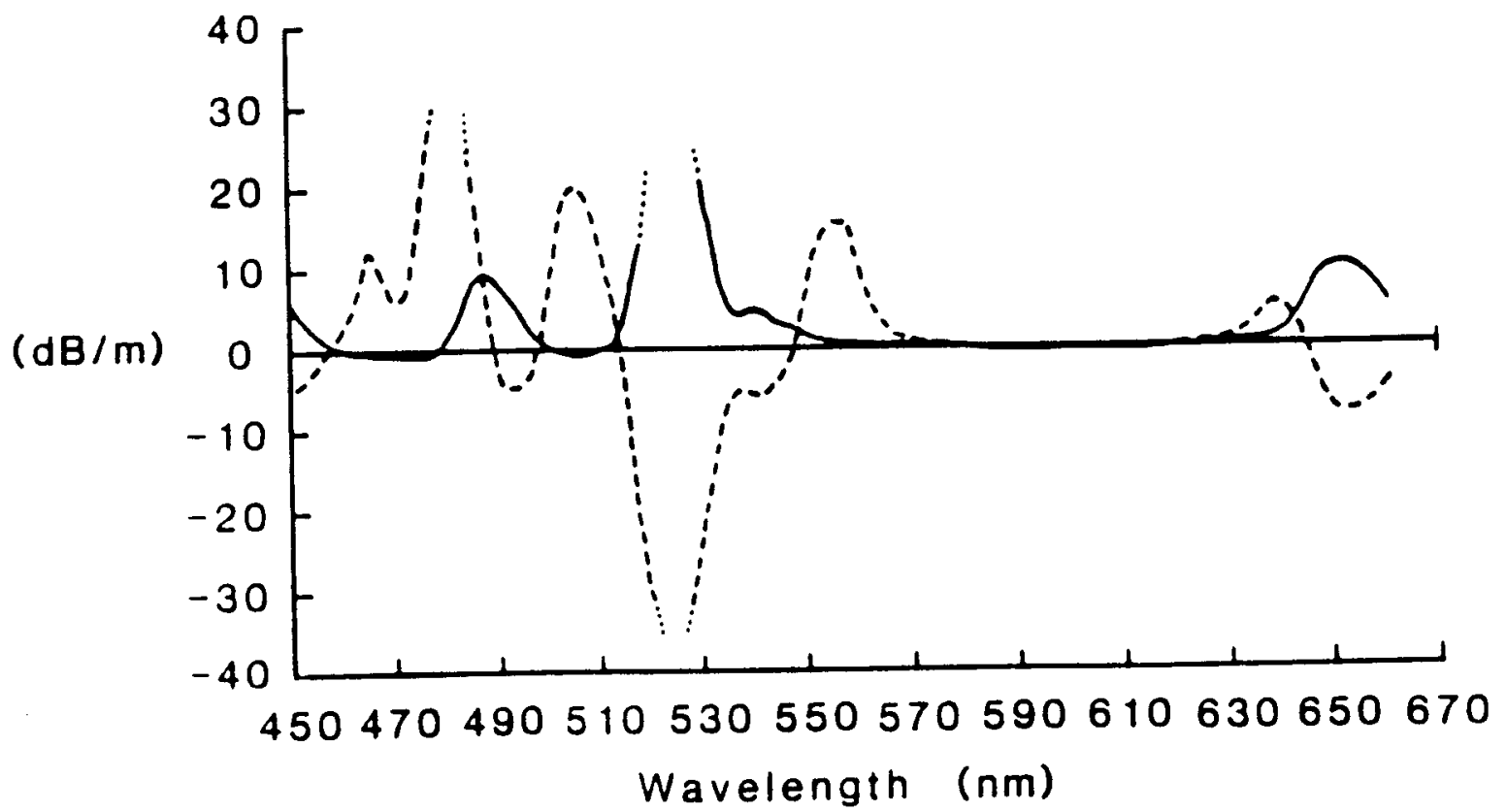

Figure 8. ESA and GSA spectra of $\mathrm{Er}^{3+}$ doped aluminosilicate core fibre 


\begin{tabular}{|l|c|c|c|c|}
\hline $\begin{array}{l}\text { Fibre TYpe } \\
\text { Wavelength (nm) }\end{array}$ & $\mathrm{GeO}_{2}$ & $\mathrm{GeO}_{2} / \mathrm{B}_{2} \mathrm{O}_{3}$ & $\mathrm{GeO}_{2} / \mathrm{P}_{2} \mathrm{O}_{5}$ & $\mathrm{Al}_{2} \mathrm{O}_{3}$ \\
\hline 488 & 2.9 & & 1.86 & 1.74 \\
\hline 514.5 & 0.95 & & 0.55 & 0.5 \\
\hline 655 & 0.28 & 0.25 & 0.13 & 0.14 \\
\hline 810 & 2.0 & 2.0 & 1.0 & 1.0 \\
\hline 980 & & & & 0 \\
\hline
\end{tabular}

Table 1. Ratio of ESA and GSA absorption cross sections, $\sigma_{\mathrm{ESA}} / \sigma_{\mathrm{GSA}}$ as a function of wavelength (after Laming et a1) 